\title{
Applications of the Rådström-Hörmander Embedding Theorem to Multifunctions
}

by

\author{
Anna KUCIA
}

\author{
Presented by Czestaw OLECH
}

Summary. Using the Rådström-Hörmander theorem on embedding of the hyperspace of closed convex sets in a Banach space, we prove multivalued versions of some results known for real functions.

1. Notation and basic facts. Throughout the paper $X$ will denote a topological space and $Y$ a metric space with a metric $\varrho$ or a normed linear space with $\varrho(x, y)=\|x-y\|$. The open (resp., closed) $\varepsilon$-ball in $Y$ around a nonempty set $A \subset Y$ is denoted by $B(A, \varepsilon)=\{y \in Y: \varrho(y, A)<\varepsilon\}$ $=\bigcup\{B(a, \varepsilon): a \in A\}$ (resp., $\bar{B}(A, \varepsilon)=\{y \in Y: \varrho(y, A) \leq \varepsilon\}$ ), where $\varrho(y, A)=\inf \{\varrho(y, a): a \in A\}$. Clearly $\operatorname{cl} B(A, \varepsilon) \subset \bar{B}(A, \varepsilon)$, where $\operatorname{cl} F$ or $\operatorname{cl}_{Y} F$ denotes the closure of $F$ in $Y$.

We denote by $2^{Y}$ the family of all nonempty closed subsets of $Y$. We shall consider the following subfamilies of $2^{Y}$ :

$$
\begin{aligned}
& \mathcal{F}(Y)=\left\{A \in 2^{Y}: A \text { is bounded }\right\} \\
& \mathcal{K}(Y)=\{A \in \mathcal{F}(Y): A \text { is compact }\}
\end{aligned}
$$

and in the case of a normed space $Y$ :

$$
\begin{aligned}
2_{\mathrm{c}}^{Y} & =\left\{A \in 2^{X}: A \text { is convex }\right\}, \\
\mathcal{F}_{\mathrm{c}}(Y) & =\{A \in \mathcal{F}(Y): A \text { is convex }\}, \\
\mathcal{K}_{\mathrm{c}}(Y) & =\{A \in \mathcal{K}(Y): A \text { is convex }\} .
\end{aligned}
$$

It is known that any subset $\mathcal{A} \subset 2^{Y}$ can be regarded as a (generalized) metric space with the Hausdorff distance $\operatorname{dist}(A, C)=\inf \{\varepsilon>0: A \subset$

2000 Mathematics Subject Classification: 26E25, 54C60.

Key words and phrases: Hausdorff continuous multifunction, convex values, extension, sandwich theorem, monotone approximation. 
$B(C, \varepsilon)$ and $C \subset B(A, \varepsilon)\}$. Balls in the Hausdorff metric will be denoted by $\mathcal{B}(A, \varepsilon)$.

If the space $Y$ is complete, then $(\mathcal{F}(Y)$, dist) is also complete (a result due to $\mathrm{H}$. Hahn, 1932) and $\mathcal{K}(Y)$ is a closed subset of $\mathcal{F}(Y)$. If $Y$ is compact, then so is $\mathcal{K}(Y)$. It is easy to see that $2_{\text {c }}^{Y}$ is a closed subset of $2^{Y}$ whenever $Y$ is normed. Thus, if $F$ is a complete (resp., compact) subset of a normed space $Y$, then $\mathcal{F}_{\mathrm{c}}(F)$ is also a complete (resp., compact) subset of $\mathcal{F}_{\mathrm{c}}(Y)$. If $Y$ is a Banach space, then the spaces $\left(\mathcal{F}_{\mathrm{c}}(Y)\right.$, dist $)$ and $\left(\mathcal{K}_{\mathrm{c}}(Y)\right.$, dist $)$ are complete. If $Y$ is separable (resp., separable and normed), then $\mathcal{K}(Y)$ (resp., $\mathcal{K}_{\mathrm{c}}(Y)$ ) is also separable (cf. e.g. [7; 4.5.22] and [2; Ch. II]).

Let $Y$ be normed and let $\mathcal{A}$ be $2_{\mathrm{c}}^{Y}, \mathcal{F}_{\mathrm{c}}(Y)$ or $\mathcal{K}_{\mathrm{c}}(Y)$. For $A, B \in \mathcal{A}$ and $\lambda \in[0, \infty)$ we define: $A+B=\{a+b: a \in A, b \in B\}, A \dot{+} B=\operatorname{cl}(A+B)$, and $\lambda A=\{\lambda a: a \in A\}$. Clearly, $A+B$ and $\lambda A$ belong to $\mathcal{A}$. Moreover, $A \dot{+} B=A+B$ whenever $A, B \in \mathcal{K}_{\mathrm{c}}(Y)$. As usual, a set $\mathcal{C} \subset \mathcal{A}$ is called convex if $\alpha A \dot{+} \beta B \in \mathcal{C}$ for every $A, B \in \mathcal{C}, \alpha+\beta=1, \alpha, \beta \geq 0$. For example, $\{A \in \mathcal{A}: A \subset F\}$ is closed (resp., compact) and convex in $\mathcal{A}$ if $F \subset Y$ is closed (resp., compact) and convex.

By a multifunction we mean any set-valued function. Let $X$ be a topological space, $Y$ a (generalized) metric space, and $\varphi: X \rightarrow 2^{Y}$ a multifunction. By $\varphi(X)$ we denote $\bigcup\{\varphi(x): x \in X\}$. We say that $\varphi$ is lower (resp., upper) semicontinuous if for each open (resp., closed) $A \subset Y$ the preimage $\varphi^{-}(A)=\{x \in X: \varphi(x) \cap A \neq \emptyset\}$ is open (resp., closed) in $X$. We shall use the abbreviations l.s.c. and u.s.c. A multifunction $\varphi$ is said to be Hausdorff lower semicontinuous (resp., Hausdorff upper semicontinuous), for short H.l.s.c. (resp., H.u.s.c), if for each $x_{0} \in X$ and $\varepsilon>0$ there exists a neighbourhood $U$ of $x_{0}$ such that $\varphi\left(x_{0}\right) \subset B(\varphi(x), \varepsilon)$ (resp., $\left.\varphi(x) \subset B\left(\varphi\left(x_{0}\right), \varepsilon\right)\right)$ whenever $x \in U$. Recall that any H.l.s.c. multifunction is l.s.c. and any u.s.c. multifunction is H.u.s.c. In the case of compact-valued multifunctions the corresponding notions coincide. If $\varphi$ is H.l.s.c. and H.u.s.c. (resp., l.s.c. and u.s.c.), then it is called $H$-continuous (resp., V-continuous). Observe that a multifunction $\varphi: X \rightarrow \mathcal{A}$ with $\mathcal{A} \subset 2^{Y}$ is H-continuous (resp., $\mathrm{V}$-continuous) if and only if $\varphi$ considered as a function from $X$ to the space $\mathcal{A}$ with the Hausdorff distance (the Vietoris topology) is continuous.

It is known that generally $\mathrm{H}$-continuity is not preserved under finite intersections. Usually, to obtain such a result, we assume that the interior of the intersection is nonempty at every point. The following proposition, perhaps known, is similar (cf. [5; Lemma 2.2 and Prop. 2.3], [10; Lemma 2.1] and [12]).

Proposition. Let $\varphi_{0}: X \rightarrow \mathcal{F}_{\mathrm{c}}(Y)$ and $\varphi_{i}: X \rightarrow 2_{\mathrm{c}}^{Y}, i=1, \ldots, n$, be H-continuous (resp., locally H-Lipschitzean) multifunctions, where $Y$ is normed (and $X$ metric, resp.). If $r_{i}>0, i=1, \ldots, n$, and $\bigcap_{i=0}^{n} \varphi_{i}(x) \neq \emptyset$ 
for every $x \in X$, then $\psi: X \rightarrow \mathcal{F}_{\mathrm{c}}(Y)$ defined by

$$
\psi(x)=\varphi_{0}(x) \cap \bigcap_{i=1}^{n} \bar{B}\left(\varphi_{i}(x), r_{i}\right)
$$

is H-continuous (resp., locally H-Lipschitzean).

Proof. First we claim the following: If $F \subset Y$ and $G_{\lambda} \subset Y, \lambda \in \Lambda$, are convex, $F$ is bounded with $\operatorname{diam}(F) \leq M$, and $B\left(y_{0}, r\right) \subset G_{\lambda}$ for some $y_{0} \in F, r>0$ and every $\lambda \in \Lambda$, then for each $\delta>0$ the following inclusion holds:

$$
B(F, \delta) \cap \bigcap_{\lambda \in \Lambda} B\left(G_{\lambda}, \delta\right) \subset B\left(F \cap \bigcap_{\lambda \in \Lambda} G_{\lambda}, \delta\left(1+\frac{2 M}{r}\right)\right) .
$$

Indeed, for $y \in B(F, \delta) \cap \bigcap_{\lambda \in \Lambda} B\left(G_{\lambda}, \delta\right)$ we take $y^{\prime} \in F$ and $y_{\lambda} \in G_{\lambda}$ such that $\left\|y-y^{\prime}\right\|<\delta$ and $\left\|y-y_{\lambda}\right\|<\delta$. Then

$$
z_{\lambda}=y_{0}+\frac{r}{2 \delta}\left(y^{\prime}-y_{\lambda}\right) \in B\left(y_{0}, r\right) \subset G_{\lambda} .
$$

We put

$$
z=\frac{2 \delta}{r+2 \delta} y_{0}+\frac{r}{r+2 \delta} y^{\prime}=\frac{2 \delta}{r+2 \delta} z_{\lambda}+\frac{r}{r+2 \delta} y_{\lambda} .
$$

Hence $z \in F \cap \bigcap_{\lambda \in \Lambda} G_{\lambda}, F$ and $G_{\lambda}$ being convex and $y_{0}, y^{\prime} \in F, z_{\lambda}, y_{\lambda} \in G_{\lambda}$. Moreover,

$$
\|y-z\| \leq\left\|y-y^{\prime}\right\|+\left\|y^{\prime}-z\right\| \leq \delta+\left\|\frac{2 \delta}{r+2 \delta}\left(y_{0}-y^{\prime}\right)\right\|<\delta\left(1+\frac{2 M}{r}\right) .
$$

This proves our claim.

Let $x_{0} \in X$. Since $\varphi_{0}\left(x_{0}\right)$ is bounded and $\varphi_{0}$ is H.u.s.c. at $x_{0}$, there exist $M>0$ and a neighbourhood $V$ of $x_{0}$ such that $\operatorname{diam}\left(\varphi_{0}(x)\right) \leq M$ for $x \in V$. If $\varphi_{0}, \ldots, \varphi_{n}$ are H-continuous at $x_{0}$, then for given $\varepsilon>0$ we take a neighbourhood $U \subset V$ of $x_{0}$ such that $\operatorname{dist}\left(\varphi_{i}(x), \varphi_{i}\left(x_{0}\right)\right)<\delta^{\prime}$ for $x \in U$ and $i=0, \ldots, n$, where $\delta=\varepsilon /(1+2 M / r), r=\min \left\{r_{1}, \ldots, r_{n}\right\}$ and $0<\delta^{\prime}<\delta$. So for every $x \in U$, by the first part of the proof, we obtain

$$
\begin{aligned}
\psi\left(x_{0}\right) & \subset B\left(\varphi_{0}(x), \delta^{\prime}\right) \cap \bigcap_{i=1}^{n} B\left(\bar{B}\left(\varphi_{i}(x), r_{i}\right), \delta^{\prime}\right) \\
& \subset B(\psi(x), \delta(1+2 M / r))=B(\psi(x), \varepsilon),
\end{aligned}
$$

and analogously, $\psi(x) \subset B\left(\psi\left(x_{0}\right), \varepsilon\right)$. This proves the H-continuity of $\psi$ at $x_{0}$.

If we consider H-locally Lipschitzean multifunctions, we take $U \subset V$ such that $\varphi_{i} \mid U$ is H-Lipschitzean with constant $k_{i}, i=0, \ldots, n$. Then for $k>k^{\prime}>\max \left\{k_{0}, \ldots, k_{n}\right\}$ and any $x, y \in U, x \neq y$, we have 


$$
\begin{aligned}
\psi(y) & \subset B\left(\varphi_{0}(x), k^{\prime} \varrho(x, y)\right) \cap \bigcap_{i=1}^{n} B\left(\bar{B}\left(\varphi_{i}(x), r_{i}\right), k^{\prime} \varrho(x, y)\right) \\
& \subset B(\psi(x), k(1+2 M / r) \varrho(x, y)) .
\end{aligned}
$$

This shows that $\psi \mid U$ is H-Lipschitzean.

Note that in the following lemma all multifunctions $\Phi$ are of the type $\Phi: X \rightarrow 2^{\mathcal{A}}$, i.e., the values $\Phi(x)$ are closed subsets of the space $\mathcal{A}$ endowed with the Hausdorff distance.

Lemma. Assume that $X$ is a topological space, $Y$ a metric (resp., normed) space, $\mathcal{A}$ one of the spaces $2^{Y}, \mathcal{F}(Y)$ or $\mathcal{K}(Y)$ (resp., $2_{\mathrm{c}}^{Y}, \mathcal{F}_{\mathrm{c}}(Y)$ or $\mathcal{K}_{\mathrm{c}}(Y)$ ) and $x_{0} \in X$. Then for any multifunctions $\varphi: X \rightarrow \mathcal{A}$ and $\psi: X \rightarrow 2^{Y}$ (resp., $\psi: X \rightarrow 2_{\mathrm{c}}^{Y}$ ), the following hold:

(a) If $\Phi(x)=\{A \in \mathcal{A}: A \subset \psi(x)\}$ for $x \in X$, then $\Phi: X \rightarrow 2^{\mathcal{A}}$ (resp., $\left.\Phi: X \rightarrow 2_{\mathrm{c}}^{\mathcal{A}}\right)$ and

(1) $\psi$ is H.l.s.c. at $x_{0}$ if and only if $\Phi$ is H.l.s.c. at $x_{0}$,

(2) $\psi$ is H.u.s.c. at $x_{0}$ if and only if $\Phi$ is H.u.s.c. at $x_{0}$.

(b) If $\Phi(x)=\{A \in \mathcal{A}: \varphi(x) \subset A\}$ for $x \in X$, then $\Phi: X \rightarrow 2^{\mathcal{A}}$ (resp., $\left.\Phi: X \rightarrow 2_{\mathrm{c}}^{\mathcal{A}}\right)$ and

(3) $\varphi$ is H.l.s.c. at $x_{0}$ if and only if $\Phi$ is H.u.s.c. at $x_{0}$,

(4) $\varphi$ is H.u.s.c. at $x_{0}$ if and only if $\Phi$ is H.l.s.c. at $x_{0}$.

(c) If $\varphi(x) \subset \psi(x)$ and $\Phi(x)=\{A \in \mathcal{A}: \varphi(x) \subset A \subset \psi(x)\}$ for all $x \in X$, then $\Phi: X \rightarrow 2^{\mathcal{A}}$ (resp., $\Phi: X \rightarrow 2_{\mathrm{c}}^{\mathcal{A}}$ ) and

(5) $\varphi$ is H.u.s.c. at $x_{0}$ and $\psi$ is H.l.s.c. at $x_{0}$ if and only if $\Phi$ is H.l.s.c. at $x_{0}$,

(6) $\varphi$ is H.l.s.c. at $x_{0}$ and $\psi$ is H.u.s.c. at $x_{0}$ if and only if $\Phi$ is H.u.s.c. at $x_{0}$.

Proof. Obviously, (b) is a consequence of (c) with $\psi(x)=Y$ for $x \in X$. Since $\varphi(x) \neq \emptyset$, (a) is not a special case of (c), but the proof of (c) given below works also under the assumption $\varphi(x)=\emptyset$ for each $x \in X$.

Observe that $\varphi(x) \in \Phi(x)$ under (b) or (c), and $\{y\} \in \Phi(x)$ for $y \in \psi(x)$ under (a), hence $\Phi(x) \neq \emptyset$. Since $\varphi(x)$ and $\psi(x)$ are closed (resp., closed and convex) subsets of $Y, \Phi(x)$ is a nonempty closed (resp., closed and convex) subset of $\mathcal{A}$, i.e., $\Phi(x) \in 2^{\mathcal{A}}$ (resp., $\Phi(x) \in 2_{\mathrm{c}}^{\mathcal{A}}$ ).

In order to prove (5), assume first that $\varphi$ is H.u.s.c. at $x_{0}$ and $\psi$ is H.l.s.c. at $x_{0}$. We have to find for each $\varepsilon>0$ a neighbourhood $U$ of $x_{0}$ such that $\Phi\left(x_{0}\right) \subset \mathcal{B}(\Phi(x), \varepsilon)$ for every $x \in U$, i.e., such that if $A_{0} \in \mathcal{A}, x \in U$ and $\varphi\left(x_{0}\right) \subset A_{0} \subset \psi\left(x_{0}\right)$, then

$$
\operatorname{dist}\left(A_{x}, A_{0}\right)<\varepsilon \quad \text { for some } A_{x} \in \mathcal{A} \text { with } \varphi(x) \subset A_{x} \subset \psi(x) .
$$


Let $U$ be a neighbourhood of $x_{0}$ such that $\varphi(x) \subset B\left(\varphi\left(x_{0}\right), \varepsilon / 2\right)$ and $\psi\left(x_{0}\right) \subset$ $B(\psi(x), \varepsilon / 2)$ for $x \in U$. Fix $x \in U$ and $A_{0} \in \Phi\left(x_{0}\right)$.

If $\mathcal{A}=2^{Y}$ or $\mathcal{A}=\mathcal{F}(Y)\left(\mathcal{A}=2_{\mathrm{c}}^{Y}\right.$ or $\left.\mathcal{A}=\mathcal{F}_{\mathrm{c}}(Y)\right)$ we take $A_{x}=$ $\psi(x) \cap \bar{B}\left(A_{0}, \varepsilon / 2\right)$. Clearly, $A_{x}$ is closed (resp., closed and convex) and $\varphi(x) \subset$ $A_{x} \subset \psi(x)$, because $\varphi(x) \subset B\left(\varphi\left(x_{0}\right), \varepsilon / 2\right) \subset B\left(A_{0}, \varepsilon / 2\right)$. For each $y_{0} \in A_{0} \subset$ $\psi\left(x_{0}\right) \subset B(\psi(x), \varepsilon / 2)$ there exists $y \in \psi(x)$ such that $\varrho\left(y, y_{0}\right)<\varepsilon / 2$, i.e., $y \in A_{x}$ and consequently $A_{0} \subset B\left(A_{x}, \varepsilon / 2\right)$. (This shows that $A_{x} \neq \emptyset$ also for $\varphi \equiv \emptyset$, i.e., in the proof of $(1))$. Hence, $\operatorname{dist}\left(A_{x}, A_{0}\right) \leq \varepsilon / 2<\varepsilon$ and $A_{x} \in \Phi(x)$.

In the case of $\mathcal{A}=\mathcal{K}(Y)$ (resp., $\mathcal{A}=\mathcal{K}_{\mathrm{c}}(Y)$ ), we proceed in the following way. Since $A_{0} \subset \psi\left(x_{0}\right) \subset B(\psi(x), \varepsilon / 2)$ is compact, there exist $k \in \mathbb{N}$, $a_{i} \in A_{0}$ and $b_{i} \in \psi(x), i=1, \ldots, k$, such that $\varrho\left(y_{0},\left\{a_{1}, \ldots, a_{k}\right\}\right)<\varepsilon / 2$ for every $y_{0} \in A_{0}$ and $\varrho\left(b_{i}, a_{i}\right)<\varepsilon / 2$. We put $A_{x}=\varphi(x) \cup\left\{b_{1}, \ldots, b_{k}\right\}$ (resp., $A_{x}=\operatorname{conv}\left(\varphi(x) \cup\left\{b_{1}, \ldots, b_{k}\right\}\right)$ ). Then $A_{x} \in \mathcal{A}(\varphi(x)$ being convex and compact), $\varphi(x) \subset A_{x} \subset \psi(x)\left(\psi(x)\right.$ being convex) and $A_{0} \subset$ $B\left(\left\{b_{1}, \ldots, b_{k}\right\}, \varepsilon\right) \subset B\left(A_{x}, \varepsilon\right)$. On the other hand, $\left\{b_{1}, \ldots, b_{k}\right\} \subset B\left(A_{0}, \varepsilon / 2\right)$ and $\varphi(x) \subset B\left(\varphi\left(x_{0}\right), \varepsilon / 2\right) \subset B\left(A_{0}, \varepsilon / 2\right)$, so $A_{x} \subset B\left(A_{0}, \varepsilon / 2\right)\left(A_{0}\right.$ being convex). This shows that $\operatorname{dist}\left(A_{0}, A_{x}\right)<\varepsilon$.

To prove the converse implication in (5) we take a neighbourhood $U$ of $x_{0}$ such that $\Phi\left(x_{0}\right) \subset \mathcal{B}(\Phi(x), \varepsilon)$ for $x \in U$. If $x \in U$, then $A_{0}=\left\{y_{0}\right\} \cup \varphi\left(x_{0}\right)$ (resp., $A_{0}=\operatorname{cl} \operatorname{conv}\left(\varphi\left(x_{0}\right) \cup\left\{y_{0}\right\}\right)$ ) belongs to $\Phi\left(x_{0}\right)$ for any $y_{0} \in \psi\left(x_{0}\right)$. Hence, $\operatorname{dist}\left(A_{0}, A_{x}\right)<\varepsilon$ for some $A_{x} \in \Phi(x)$ and $y_{0} \in A_{0} \subset B\left(A_{x}, \varepsilon\right) \subset$ $B(\psi(x), \varepsilon)$. Consequently, $\psi\left(x_{0}\right) \subset B(\psi(x), \varepsilon)$ for $x \in U$, i.e., $\psi$ is H.l.s.c. at $x_{0}$. Since $\varphi\left(x_{0}\right) \in \Phi\left(x_{0}\right)$, for every $x \in U$ there exists $A_{x} \in \Phi(x)$ such that $\operatorname{dist}\left(A_{x}, \varphi\left(x_{0}\right)\right)<\varepsilon$. Hence, $\varphi(x) \subset A_{x} \subset B\left(\varphi\left(x_{0}\right), \varepsilon\right)$ for $x \in U$, i.e., $\varphi$ is H.u.s.c. at $x_{0}$.

The proof of (6) is the same as that of (5) with $A_{0}$ replacing $A_{x}$ and vice versa.

\section{Applications of the Rådström-Hörmander Theorem. Recall} the following version of the Rådström-Hörmander Theorem:

TheOREm. Let $Y$ be a normed space and $\mathcal{A}=\mathcal{F}_{\mathrm{c}}(Y)$ or $\mathcal{A}=\mathcal{K}_{\mathrm{c}}(Y)$. Then the space $(\mathcal{A}$, dist $)$ with the operations $(A, B) \mapsto A \dot{+} B$ and $(\lambda, A) \mapsto$ $\lambda A, A, B \in \mathcal{A}, \lambda \in[0, \infty)$, can be isometrically and algebraically embedded as a convex cone $K$ in a Banach space $E$.

In 1952 the above theorem was proved by Rådström [14] for some $\mathcal{A} \subset$ $\mathcal{F}_{\mathrm{c}}(Y)$ with the operation + (e.g. for $\mathcal{K}_{\mathrm{c}}(Y)$ and for $\left\{A \in \mathcal{K}_{\mathrm{c}}(Y): A\right.$ is finitedimensional $\}$ ). However, due to the cancellation law for the operation $\dot{+}$ (see e.g. [17; Prop. 2.1]), his proof also works in the case of $\left(\mathcal{F}_{\mathrm{c}}(Y), \dot{+}\right)$. The proof for $\left(\mathcal{F}_{\mathrm{c}}(Y), \dot{+}\right)$ using support functions was given by Hörmander [11] in 1954 .

In what follows, for the sake of simplicity, we identify $\mathcal{A}$ with the convex cone $K \subset E$. Observe that if $F \subset Y$ is complete and $\mathcal{A}=\mathcal{F}_{\mathrm{c}}(Y)$, then $\mathcal{F}_{\mathrm{c}}(F)$ 
is also a complete subset of $E$, since the embedding is an isometry. Similarly, if $F \subset Y$ is compact and $\mathcal{A}=\mathcal{F}_{\mathrm{c}}(Y)$ or $\mathcal{A}=\mathcal{K}_{\mathrm{c}}(Y)$, then $\mathcal{K}_{\mathrm{c}}(F)$ is a compact subset of $E$. In particular, if in the Rådström-Hörmander Theorem $Y$ is assumed to be a Banach space, then the cone $K$ is a closed subset of $E$, since the spaces $\left(\mathcal{F}_{\mathrm{c}}(Y)\right.$, dist) and $\left(\mathcal{K}_{\mathrm{c}}(Y)\right.$, dist $)$ are complete. If $Y$ is separable and $\mathcal{A}=\mathcal{K}_{\mathrm{c}}(Y)$, then one can assume that $E$ is also separable.

From the Rådström-Hörmander Theorem one can obtain some interesting results for multifunctions, as corollaries to known theorems. By the Dugundji Extension Theorem (e.g. [6; Th. 10.4]) we immediately obtain the following result:

Corollary 1 (Extension Theorem). Let $Y$ be a normed space and $\mathcal{A}=$ $\mathcal{F}_{\mathrm{c}}(Y)\left(\mathcal{A}=\mathcal{K}_{\mathrm{c}}(Y)\right.$ or $\mathcal{A}=\left\{A \in \mathcal{K}_{\mathrm{c}}(Y): A\right.$ is finite-dimensional $\left.\}\right)$. Then any H-continuous multifunction $\varphi: F \rightarrow \mathcal{A}$ defined on a closed subset $F$ of a metrizable space $X$ has an $H$-continuous extension $\bar{\varphi}: X \rightarrow \mathcal{A}$ such that $\bar{\varphi}(X) \subset \operatorname{clconv} \varphi(F)(r e s p ., \bar{\varphi}(X) \subset \operatorname{conv} \varphi(F))$.

Proof. We treat $\varphi$ as a continuous function $\varphi: F \rightarrow K \subset E$. Hence, there exists a continuous extension $\bar{\varphi}: X \rightarrow E$ such that $\bar{\varphi}(x) \in \operatorname{conv}_{E}\{\varphi(x)$ : $x \in F\} \subset K$ for $x \in X$, i.e., if $\bar{\varphi}$ is regarded as a multifunction, then it is H-continuous, $\bar{\varphi}(x) \in \mathcal{A}$ and $\bar{\varphi}(x) \subset \operatorname{clconv} \varphi(F)$ (resp., $\bar{\varphi}(x) \subset \operatorname{conv} \varphi(F)$, since for compact sets the operations + and $\dot{+}$ coincide $)$.

REMARK. In the same way one can also obtain the following results: Let $\mathcal{A}$ be $\mathcal{F}_{\mathrm{c}}(Y)$ or $\mathcal{K}_{\mathrm{c}}(Y)$. A $\mathrm{T}_{1}$-space $X$ is collectionwise normal iff for every Banach space $Y$ and every closed $F \subset X$ any H-continuous $\varphi: F \rightarrow$ $\mathcal{A}$ can be extended to an H-continuous $\bar{\varphi}: X \rightarrow \mathcal{A}$ such that $\bar{\varphi}(X) \subset$ clconv $\varphi(F)$. If we restrict ourselves to separable Banach spaces and $\mathcal{A}=$ $\mathcal{K}_{\mathrm{c}}(Y)$, then we obtain a corresponding characterization of normal spaces. It follows, respectively, from theorems due to Dowker and Hanner (cf. also [13; Th. 3.2 and Th. 3.1]), and from the observation that the set $\{A \in \mathcal{A}: A \subset$ clconv $\varphi(F)\}$ is a retract of $E$, being a closed and convex subset of $E$.

It is known that some extension theorems can be deduced from selection theorems (cf. [13; Cor. 1.5]). For example, the Michael Selection Theorem [13; Th. 3.2" $]$ yields the following result:

Corollary 2 (Extension Theorem). Let $Y$ be a Banach space and $\mathcal{A}=$ $\mathcal{F}_{\mathrm{c}}(Y)$ or $\mathcal{A}=\mathcal{K}_{\mathrm{c}}(Y)$. Then any H-continuous multifunction $\varphi: F \rightarrow \mathcal{A}$ defined on a closed subset $F$ of a paracompact space $X$ has an $H$-continuous extension $\bar{\varphi}: X \rightarrow \mathcal{A}$ such that $\bar{\varphi}(X) \subset$ clconv $\varphi(F)$. Moreover, if $\psi: X \rightarrow$ $2_{\mathrm{c}}^{Y}$ is H.l.s.c. and $\varphi(x) \subset \psi(x)$ for $x \in F$, then there exists an H-continuous extension $\bar{\varphi}: X \rightarrow \mathcal{A}$ such that $\bar{\varphi}(x) \subset \psi(x)$ for each $x \in X$.

Proof. If $\psi$ is not given, then we put $\psi(x)=\operatorname{clconv} \varphi(F)$ for $x \in X$. By (1) of the Lemma, the multifunction $\Phi: X \rightarrow 2_{\mathrm{c}}^{\mathcal{A}}, \Phi(x)=\{A \in \mathcal{A}$ : 
$A \subset \psi(x)\}$, can be regarded as an H.l.s.c. multifunction from $X$ to a Banach space $E$ with closed and convex values. Hence $\Psi$ defined by $\Psi(x)=\{\varphi(x)\}$ for $x \in F$ and $\Psi(x)=\Phi(x)$ for $x \in X \backslash F$ is l.s.c., $F$ being closed and $\varphi$ $\mathrm{H}$-continuous. Every continuous selection of $\Psi$, the existence of which follows by the Michael Theorem, is the desired extension.

REMARKS. 1. Instead of the completeness of $Y$, in the above corollary one can assume that $\psi$ has complete values.

2. If $\psi: X \rightarrow \mathcal{K}_{\mathrm{c}}(Y)$ and $Y$ is normed, it is enough to assume that $X$ is collectionwise normal; if additionally $Y$ is separable, then $X$ can be normal. This follows in the same way from selection theorems for compact-convexvalued multifunctions (cf. [13; Th. 3.2' and Th. 3.1']). The existence of such extensions for every $\varphi$ and $\psi$ characterizes, respectively, the collectionwise normality and the normality of $X$ ( $X$ is assumed to be a $\mathrm{T}_{1}$-space).

3. A result corresponding to Corollary 2 in the case of a metric space $X$ and H-continuous $\psi: X \rightarrow \mathcal{K}_{\mathrm{c}}(Y)$ was given in [16]. Its proof is based on support functions.

Corollary 3 (Sandwich Theorem). Let $X$ be paracompact, $Y$ a Banach space and $\mathcal{A}=\mathcal{F}_{\mathrm{c}}(Y)$ or $\mathcal{A}=\mathcal{K}_{\mathrm{c}}(Y)$. Then for any H.u.s.c. multifunction $\varphi: X \rightarrow \mathcal{A}$ and any H.l.s.c. $\psi: X \rightarrow 2_{\mathrm{c}}^{Y}$ such that $\varphi(x) \subset \psi(x)$ for all $x \in X$, there exists an H-continuous multifunction $\chi: X \rightarrow \mathcal{A}$ such that $\varphi(x) \subset$ $\chi(x) \subset \psi(x)$ for each $x \in X$. In particular, any H.u.s.c. multifunction $\varphi$ : $X \rightarrow \mathcal{A}$ is bounded by an $H$-continuous $\chi: X \rightarrow \mathcal{A}$.

Proof. Let $\Phi(x)=\{A \in \mathcal{A}: \varphi(x) \subset A \subset \psi(x)\}$. By (5) of the Lemma, $\Phi$ is a H.l.s.c. multifunction with closed and convex values from $X$ to a Banach space $E$. Hence it has a continuous selection $\chi$.

REmark. The above result in the case of a metric space $X$ and $\varphi, \psi$ : $X \rightarrow \mathcal{K}_{\mathrm{c}}\left(\mathbb{R}^{n}\right)$ was proved in [1; Th. 4]. A related result for a metric space $X$ and $\varphi, \psi: X \rightarrow \mathcal{F}_{\mathrm{c}}(Y)$ such that $B(\varphi(x), r(x)) \subset \psi(x)$ for some $r(x)>0$, $x \in X$, was proved in [4; Th. 5.1]. Similar results (called interposition theorems) were announced in [8] and [9], but without proofs. A version of the Sandwich Theorem with $\varphi$ and $\psi$ convex-compact-valued is given in the book [15; Th. 5.75], and the authors suggest another method of proof.

The following two corollaries are generalizations of known characterizations of some topological spaces by semicontinuous real functions (see e.g. $[7 ; 1.7 .15(\mathrm{~b})$ and (c)]).

Corollary 4. A $\mathrm{T}_{1}$-space $X$ is normal if and only if for every separable normed space $Y$ and $\varphi, \psi: X \rightarrow \mathcal{K}_{\mathrm{c}}(Y)$, where $\varphi$ is H.u.s.c., $\psi$ is H.l.s.c. and $\varphi(x) \subset \psi(x)$ for each $x \in X$, there exists an H-continuous multifunction $\chi: X \rightarrow \mathcal{K}_{\mathrm{c}}(Y)$ such that $\varphi(x) \subset \chi(x) \subset \psi(x)$ for each $x \in X$. 
Proof. Now $\Phi$ defined as in the previous corollary has compact convex values in a separable Banach space $E$. Hence the existence of $\chi$ follows by [13; Th. 3.1']. For the converse we consider, for a pair of sets $F, U$, where $F$ is closed, $U$ open and $F \subset U$, multifunctions $\varphi, \psi: X \rightarrow \mathcal{K}_{\mathrm{c}}(\mathbb{R})$ defined by

$$
\varphi(x)=\left\{\begin{array}{ll}
\{0\} & \text { if } x \notin F, \\
{[0,1]} & \text { if } x \in F,
\end{array} \quad \psi(x)= \begin{cases}\{0\} & \text { if } x \notin U, \\
{[0,1]} & \text { if } x \in U .\end{cases}\right.
$$

Then $\chi(x)=[f(x), g(x)]$, where $f$ and $g$ are continuous and $g(x)=0$ if $x \notin U$ and $g(x)=1$ if $x \in F$.

Corollary 5. A $\mathrm{T}_{1}$-space $X$ is perfectly normal if and only if for every separable Banach space $Y$ and every H.u.s.c. multifunction $\varphi: X \rightarrow \mathcal{K}_{\mathrm{c}}(Y)$ there exists a sequence of $H$-continuous multifunctions $\varphi_{n}: X \rightarrow \mathcal{K}_{\mathrm{c}}(Y)$ such that $\operatorname{dist}\left(\varphi_{n}(x), \varphi(x)\right) \rightarrow 0$ and $\varphi(x) \subset \varphi_{n+1}(x) \subset \varphi_{n}(x)$ for every $x \in X$.

Proof. By (4) of the Lemma the multifunction $\Phi$ defined by $\Phi(x)=$ $\left\{A \in \mathcal{K}_{\mathrm{c}}(Y): \varphi(x) \subset A\right\}$ can be regarded as a H.l.s.c. and closed-convexvalued multifunction to a separable Banach space $E$. By [13; L. 5.2], $\Phi$ has a Michael representation, i.e., there exists a countable family $\left\{\psi_{n}: n \in \mathbb{N}\right\}$ of continuous selections of $\Phi$ (i.e., $\psi_{n}: X \rightarrow \mathcal{K}_{\mathrm{c}}(Y)$ is H-continuous) such that $\left\{\psi_{n}(x): n \in \mathbb{N}\right\}$ is dense in $\Phi(x)$ for every $x \in X$. In particular, $\operatorname{dist}\left(\psi_{k_{n}}(x), \varphi(x)\right) \rightarrow 0$ for some subsequence and $\varphi(x)=\bigcap\left\{\psi_{n}(x): n \in \mathbb{N}\right\}$. We put $\varphi_{1}=\psi_{1}$ and

$$
\varphi_{n}(x)=\varphi_{n-1}(x) \cap \bar{B}\left(\psi_{n}(x), 1 / n\right), \quad n \geq 2 .
$$

Clearly, $\varphi(x) \subset \varphi_{n+1}(x) \subset \varphi_{n}(x)$ and $\operatorname{dist}\left(\varphi_{n}(x), \varphi(x)\right) \leq \operatorname{dist}\left(\psi_{n}(x), \varphi(x)\right)$ $+1 / n$. Hence, $\operatorname{dist}\left(\varphi_{n}(x), \varphi(x)\right) \rightarrow 0$ as a decreasing sequence having a subsequence which converges to 0 . By the Proposition, $\varphi_{n}: X \rightarrow \mathcal{K}_{\mathrm{c}}(Y)$ is H-continuous.

For the converse, let $F \subset X$ be closed and let $\varphi: X \rightarrow \mathcal{K}_{\mathrm{c}}(\mathbb{R})$ be defined by

$$
\varphi(x)= \begin{cases}\{0\} & \text { if } x \notin F, \\ {[0,1]} & \text { if } x \in F .\end{cases}
$$

Consider the corresponding sequence $\left(\varphi_{n}\right)$. Since $\varphi_{n}(x)=\left[f_{n}(x), g_{n}(x)\right]$ and $\varphi_{n}$ is H-continuous, it follows that $g_{n}$ is continuous and $\left(g_{n}\right)$ is a decreasing sequence converging pointwise to the characteristic function of $F$.

REMARKS. 1. Recall that it is quite easy to obtain a result corresponding to the above corollary for H.l.s.c. multifunctions. Namely, for a given H.l.s.c. $\varphi: X \rightarrow \mathcal{K}_{\mathrm{c}}(Y)$ and a separable normed $Y$ we take a Michael representation $\left\{f_{n}: n \in \mathbb{N}\right\}$ and put $\varphi_{n}(x)=\operatorname{conv}\left\{f_{1}(x), \ldots, f_{n}(x)\right\}$. Clearly, the compactness of $\varphi(x)$ is essential, since the $\varphi_{n}(x)$ are compact. A related characterization of perfectly normal domains by approximation of lower semicontinuous multifunctions was given in [3]. 
2. It is easy to see that for any $X$ and $\varphi: X \rightarrow 2_{\mathrm{c}}^{Y}$ with a Michael representation, the sequence $\left(\varphi_{n}\right)$ defined as in the above remark converges pointwise to $\varphi$ in the Vietoris topology.

3. Aseev [1; Th. 2] proved a corresponding result on approximation of a H.u.s.c. $\varphi: X \rightarrow \mathcal{K}_{\mathrm{c}}\left(\mathbb{R}^{n}\right)$, where $X$ is metric, with the additional requirement $\varphi(x) \subset \operatorname{int} \varphi_{n}(x)$. To obtain such inclusions it is enough to take $\varphi_{n}^{\prime}(x)=$ $\bar{B}\left(\varphi_{n}(x), 1 / n\right), Y=\mathbb{R}^{n}$ in our proof of Corollary 5 .

There are also some results on approximation of H.u.s.c. multifunctions defined on a metric space with values in $\mathcal{F}_{\mathrm{c}}(Y)$. Usually every approximating multifunction is defined by a partition of unity from constant multifunctions. Observe that, using the Rådström-Hörmander embedding, any multifunction defined in such a way is $\mathrm{H}$-continuous. More precisely, let $\mathcal{A}$ be $\mathcal{F}_{\mathrm{c}}(Y)$ or $\mathcal{K}_{\mathrm{c}}(Y), A_{i} \in \mathcal{A}$ and $p_{i} \geq 0$ for $i=1, \ldots, n$, and

$$
A=\operatorname{cl}_{Y} \sum_{i=1}^{n} p_{i} A_{i}=p_{1} A_{1} \dot{+} \cdots \dot{+} p_{n} A_{n} \in \mathcal{A} \text {. }
$$

If we identify $\mathcal{A}$ with a subset of $E$, the set $A$ is of the form $A=\bar{\sum}_{i=1}^{n} p_{i} A_{i}$, where $\bar{\sum}$ denotes algebraic sum in $E$. Therefore, if we define a new multifunction $\varphi$ by $\varphi(x)=\operatorname{cl}_{Y} \sum_{\lambda \in \Lambda} p_{\lambda}(x) \varphi_{\lambda}(x)$, where $\left\{p_{\lambda}: \lambda \in \Lambda\right\}$ is a locally finite partition of unity and $\varphi_{\lambda}: X \rightarrow \mathcal{A}$, then $\varphi(x)=\bar{\sum}_{\lambda \in \Lambda} p_{\lambda}(x) \varphi_{\lambda}(x)$ and we obtain exactly the same theorems on the H-continuity of $\varphi$ as for continuous functions. In particular, if $\left\{p_{\lambda}: \lambda \in \Lambda\right\}$ is a locally Lipschitzean partition of unity (i.e., it is locally finite and $p_{\lambda}$ is locally Lipschitzean for every $\lambda \in \Lambda$ ) and $A_{\lambda} \in \mathcal{A}$ then $\varphi=\operatorname{cl}_{Y} \sum_{\lambda \in \Lambda} p_{\lambda}(\cdot) A_{\lambda}$ is locally H-Lipschitzean.

For completeness we give two results which are obtained in this way.

COROLlary 6 (cf. [4; Th. 4.5]). For every H.u.s.c. multifunction $\varphi$ : $X \rightarrow \mathcal{F}_{\mathrm{c}}(Y)$, where $X$ is metric and $Y$ normed, there exists a sequence of locally H-Lipschitzean multifunctions $\varphi_{n}: X \rightarrow \mathcal{F}_{\mathrm{c}}(Y)$ such that $\operatorname{dist}\left(\varphi_{n}(x), \varphi(x)\right) \rightarrow 0$ and $\varphi(x) \subset \varphi_{n+1}(x) \subset \varphi_{n}(x)$ for every $x \in X$.

Proof. For every $x \in X$ and $n \in \mathbb{N}$ we take $\delta(x, n)$ such that $\delta(x, n)<$ $1 / n$ and $\varphi(y) \subset B(\varphi(x), 1 / n)$ if $\varrho(x, y)<\delta(x, n)$. Let $\left\{p_{\lambda}^{n}: \lambda \in \Lambda_{n}\right\}$ be a locally Lipschitzean partition of unity subordinate to $\{B(x, \delta(x, n)): x \in X\}$ and let $x_{\lambda}^{n}$ be such that $\left(p_{\lambda}^{n}\right)^{-1}(0,1] \subset B\left(x_{\lambda}^{n}, \delta\left(x_{\lambda}^{n}, n\right)\right)$. We put

$$
\psi_{n}(x)=\mathrm{cl} \sum_{\lambda \in \Lambda_{n}} p_{\lambda}^{n}(x) \varphi\left(x_{\lambda}^{n}\right) .
$$

If $p_{\lambda}^{n}(x)>0$ then $\varphi(x) \subset B\left(\varphi\left(x_{\lambda}^{n}\right), 1 / n\right)$, hence $\varphi(x) \subset B\left(\psi_{n}(x), 1 / n\right)$ for $x \in X$ and $n \in \mathbb{N}$. On the other hand, for fixed $x$ and $\varepsilon>0$ we take $\delta>0$ such that $\varphi(y) \subset B(\varphi(x), \varepsilon)$ if $\varrho(x, y)<\delta$. If $n>1 / \delta$ and $p_{\lambda}^{n}(x)>0$, then $\varrho\left(x, x_{\lambda}^{n}\right)<\delta\left(x_{\lambda}^{n}, n\right)<1 / n<\delta$ and $\varphi\left(x_{\lambda}^{n}\right) \subset B(\varphi(x), \varepsilon)$. So $\psi_{n}(x) \subset$ 
$\bar{B}(\varphi(x), \varepsilon)$ whenever $n>1 / \delta$. This shows that $\operatorname{dist}\left(\psi_{n}(x), \varphi(x)\right) \rightarrow 0$ for every $x \in X$. Note that each multifunction $x \mapsto \bar{B}\left(\psi_{n}(x), 1 / n\right)$ is locally H-Lipschitzean. We put

$$
\varphi_{n}(x)=\bigcap_{i=1}^{n} \bar{B}\left(\psi_{i}(x), 2 / i\right) .
$$

By the Proposition, $\varphi_{n}$ is locally H-Lipschitzean. Obviously, $\varphi(x) \subset \varphi_{n+1}(x)$ $\subset \varphi_{n}(x) \subset \bar{B}\left(\psi_{n}(x), 2 / n\right)$ and $\operatorname{dist}\left(\varphi(x), \varphi_{n}(x)\right) \leq \operatorname{dist}\left(\varphi(x), \psi_{n}(x)\right)+2 / n$. So $\operatorname{dist}\left(\varphi_{n}(x), \varphi(x)\right)$ tends to 0 as $n \rightarrow \infty$.

REMARKs. 1. If in the above corollary $\varphi: X \rightarrow \mathcal{K}_{\mathrm{c}}(Y)$ and $\varphi(x) \subset$ $\psi(x)$ for some H-continuous $\psi: X \rightarrow \mathcal{K}_{\mathrm{c}}(Y)$ (by Corollary 3 such a $\psi$ exists if $Y$ is Banach), then putting $\varphi_{n}(x)=\psi(x) \cap \bigcap_{i=1}^{n} \bar{B}\left(\psi_{i}(x), 2 / i\right)$, we obtain a decreasing sequence of $\mathrm{H}$-continuous compact-valued multifunctions pointwise converging to $\varphi$.

2. Repeating the first part of the previous proof for a H.l.s.c. multifunction $\psi: X \rightarrow \mathcal{A}$, where $\mathcal{A}$ is $\mathcal{F}_{\mathrm{c}}(Y)$ or $\mathcal{K}_{\mathrm{c}}(Y)$, it is easy to obtain a sequence of H-continuous (or locally H-Lipschitzean) multifunctions $\varphi_{n}: X \rightarrow \mathcal{A}$ which converges pointwise to $\psi$ and $\varphi_{n}(x) \subset \bar{B}(\psi(x), 1 / n)$ for all $x \in X$.

3 . Note that the existence of an approximation by a sequence of $\mathrm{H}$ semicontinuous multifunctions characterizes H-semicontinuity. More precisely, if a sequence of H.u.s.c. (H.l.s.c.) multifunctions $\varphi_{n}: X \rightarrow 2^{Y}$ converges pointwise to $\varphi: X \rightarrow 2^{Y}$ and for every $\varepsilon>0$ there exists $n_{0}$ such that $\varphi(x) \subset B\left(\varphi_{n}(x), \varepsilon\right)$ (resp., $\varphi_{n}(x) \subset B(\varphi(x), \varepsilon)$ ) for $n \geq n_{0}$ and $x \in X$, then $\varphi$ is H.u.s.c. (resp., H.l.s.c.). In fact, for given $x_{0}$ and $\varepsilon>0$ we fix $n$ so large that $\varphi_{n}\left(x_{0}\right) \subset B\left(\varphi\left(x_{0}\right), \varepsilon / 3\right)$ and $\varphi(x) \subset B\left(\varphi_{n}(x), \varepsilon / 3\right)$ for every $x$. We take a neighbourhood $U$ of $x_{0}$ such that $\varphi_{n}(x) \subset B\left(\varphi_{n}\left(x_{0}\right), \varepsilon / 3\right)$ for $x \in U$. It is easy to see that $\varphi(x) \subset B\left(\varphi\left(x_{0}\right), \varepsilon\right)$.

Corollary 7. Let $X$ be metric, $Y$ normed and $\mathcal{A}=\mathcal{F}_{\mathrm{c}}(Y)$ or $\mathcal{A}=\mathcal{K}_{\mathrm{c}}(Y)$. Then for every H.l.s.c. multifunction $\psi: X \rightarrow \mathcal{A}$ with complete values there exists a sequence of $H$-continuous multifunctions $\psi_{n}: X \rightarrow \mathcal{A}$ converging pointwise to $\psi$ and such that $\psi_{n}(x) \subset \psi_{n+1}(x) \subset \psi(x)$ for each $x \in X$.

Proof. First observe that if $\varphi: X \rightarrow \mathcal{A}$ is H-continuous and $\varphi(x) \subset$ $\bar{B}(\psi(x), r)$ for some $r>0$ and every $x \in X$, then for every $\varepsilon>0$ there exists an H-continuous multifunction $\chi: X \rightarrow \mathcal{A}$ such that $\chi(x) \subset \psi(x)$ and $\operatorname{dist}(\chi(x), \varphi(x)) \leq r+\varepsilon$. In fact, by (1) of the Lemma, the multifunction $\Psi$ given by $\Psi(x)=\{A \in \mathcal{A}: A \subset \psi(x)\}$ is H.l.s.c. It is easy to see that $\Psi(x) \cap \mathcal{B}(\varphi(x), r+\varepsilon)$ is never empty; for example, $\psi(x) \cap \bar{B}(\varphi(x), r+\varepsilon / 2) \neq \emptyset$ belongs to it. Define $\Phi(x)=\mathrm{cl}_{E}(\Psi(x) \cap \mathcal{B}(\varphi(x), r+\varepsilon))$. By [13; Prop. 2.5], $\Phi$ is lower semicontinuous. Since $\psi(x)$ is complete (or compact), $\Psi(x)$ is closed in $E$ and $\Phi(x) \subset \operatorname{cl}_{E} \Psi(x) \subset \mathcal{A}$. Hence, $\Phi$ has a continuous selection $\chi$, i.e., $\chi: X \rightarrow \mathcal{A}$ is H-continuous, $\chi(x) \subset \psi(x)$ and $\operatorname{dist}(\varphi(x), \chi(x)) \leq r+\varepsilon$. 
Let $\varphi_{n}: X \rightarrow \mathcal{A}$ be as in Remark 2. Let $\chi_{n}: X \rightarrow \mathcal{A}$ be H-continuous such that $\chi_{n}(x) \subset \psi(x)$ and $\operatorname{dist}\left(\varphi_{n}(x), \chi_{n}(x)\right) \leq 2 / n$, for every $x \in X$. Since $\operatorname{dist}\left(\chi_{n}(x), \psi(x)\right) \leq \operatorname{dist}\left(\varphi_{n}(x), \psi(x)\right)+2 / n$, the sequence $\left(\chi_{n}(x)\right)$ converges to $\psi(x)$. We put $\psi_{n}(x)=\operatorname{clconv}\left(\chi_{1}(x) \cup \cdots \cup \chi_{n}(x)\right)$. It is easy to check that $\left(\psi_{n}\right)$ has the desired properties.

Remark. For $\psi: X \rightarrow \mathcal{F}_{\mathrm{c}}(Y)$ with $\operatorname{int} \psi(x) \neq \emptyset$ the above result was proved in [4; Th. 3.6].

3. Some examples. The Rådström-Hörmander Theorem, which is the main tool in the previous section, is formulated for classes of bounded sets. The first example below shows that, generally, our corollaries are not true for multifunctions with unbounded values.

In the following examples $I$ denotes the interval $[0,1]$.

EXAMPLE 1. It is easy to see that no multifunction $\chi: I \rightarrow 2_{\mathrm{c}}^{\mathbb{R}}$ such that $\chi(0)=[0, \infty)$ and $\chi(1)=[a, b], a \leq b$, is H-continuous. In fact, let $f(x) \in \mathbb{R} \cup\{\infty\}$ denote the right end point of $\chi(x)$, so $f: I \rightarrow \mathbb{R} \cup\{\infty\}$. If $\chi$ is H.l.s.c. at 0 , then $f(x)=\infty$ whenever $0 \leq x<\delta$ for some $\delta>0$. Let $x_{0}=\sup \{x \in I: f(y)=\infty$ for every $y \in[0, x]\}$. If $f\left(x_{0}\right)=\infty$, then $x_{0}<1$ and $\chi$ is not H.l.s.c. at $x_{0}$. If $f\left(x_{0}\right) \in \mathbb{R}$, then $\chi$ is not H.u.s.c. at $x_{0}$. Note that this example is based on the fact that the generalized metric space $\left(2_{\mathrm{c}}^{\mathbb{R}}\right.$, dist $)$ is not connected.

This example shows that the space $\left(2_{\mathrm{c}}^{\mathbb{R}}\right.$, dist $)$ does not have the extension property for continuous functions, i.e., the restriction to bounded sets in Corollaries 1 and 2 is essential (for example, $F=\{0,1\} \subset I, \varphi(0)=[0, \infty$ ), $\varphi(1)=\{0\})$. Similarly, the Sandwich Theorem and the Approximation Theorem, i.e., Corollaries 3,6 and 7 , do not hold for unbounded sets. In fact, let $\varphi$ and $\psi$ be defined on $I$ by $\varphi(0)=[0, \infty), \varphi(x)=\{0\}$ if $x \in(0,1]$, $\psi(x)=[0, \infty)$ if $x \in[0,1)$ and $\psi(1)=\{0\}$. Then $\varphi$ is H.u.s.c., $\psi$ is H.l.s.c. and $\varphi(x) \subset \psi(x)$ for every $x \in I$, but there is no H-continuous $\chi$ between $\varphi$ and $\psi$, and $\varphi$ and $\psi$ have no approximation by sequences of $\mathrm{H}$-continuous multifunctions.

The next examples show that if we consider the problems of the previous section in the case of u.s.c. or l.s.c. multifunctions, i.e., when $\mathcal{F}_{\mathrm{c}}(Y)$ is endowed with the Vietoris topology, then the situation is quite different (cf. also Remark 2 to Cor. 5).

Example 2. Let $X$ be a normed space. The multifunction $\varphi: X \rightarrow$ $\mathcal{F}_{\mathrm{c}}(X)$ defined by $\varphi(x)=\bar{B}(x, 1)$ is H-Lipschitzean, because we have $\operatorname{dist}(\bar{B}(x, r), \bar{B}(y, r))=\|x-y\|, r \geq 0$. Obviously, $\varphi$ is also 1.s.c. Observe that $\varphi$ is u.s.c. iff $X$ is finite-dimensional. Indeed, if $X$ is infinite-dimensional, then by the Riesz Theorem there exist points $b_{n} \in \bar{B}(0,1)$ such that $\left\|b_{n}\right\|=1$ 
and $\left\|b_{n}-b_{m}\right\| \geq 1 / 2$ for $n \neq m$. Let $a_{n}=(1+1 / n) b_{n}$ and $x_{n}=(1 / n) b_{n}$. Then

$$
\frac{1}{2} \leq\left\|b_{n}-b_{m}\right\|=\left\|a_{n}-a_{m}-\frac{1}{n} b_{n}+\frac{1}{m} b_{m}\right\| \leq\left\|a_{n}-a_{m}\right\|+\frac{1}{n}+\frac{1}{m} .
$$

Hence, $1 / 4 \leq\left\|a_{n}-a_{m}\right\|$ for $m>n \geq 8$ and $F=\left\{a_{n}: n \in \mathbb{N}\right\}$ is closed, and $\bar{B}(0,1) \subset X \backslash F=U$. Clearly, $\lim x_{n}=0, a_{n} \in \varphi\left(x_{n}\right)$ and $\varphi(V) \subset U$ for no neighbourhood $V$ of 0 , i.e., $\varphi$ is not u.s.c.

EXAMPLE 3. No multifunction $\chi: I \rightarrow \mathcal{F}_{\mathrm{c}}\left(l^{1}\right)$ such that $\chi(0)=\bar{B}(0,1)$, $\chi(1)=\bar{B}(0,2)$ and $\bar{B}(0,1) \subset \chi(x)$ for every $x \in I$, is V-continuous. In fact, if $\chi$ is l.s.c., then the set $\{x \in I: \chi(x) \subset \bar{B}(0,1)\}$ is closed. Hence, $x_{0}=\sup \{x \in I: \chi(x) \subset \bar{B}(0,1)\} \in[0,1)$ and $\chi\left(x_{0}\right)=\bar{B}(0,1)$. We take a decreasing sequence $\left(x_{n}\right)$ converging to $x_{0}$ and $y_{n} \in \chi\left(x_{n}\right)$ such that $1<$ $\left\|y_{n}\right\| \leq 2$. Put $\alpha_{n}=1$ if $y_{n}(n) \geq 0$ and $\alpha_{n}=-1$ if $y_{n}(n)<0$. Let

$$
a_{n}=\frac{1}{n} y_{n}+\left(1-\frac{1}{n}\right) \alpha_{n} e_{n},
$$

where $e_{n} \in l^{1}$ is defined by $e_{n}(k)=\delta_{n}^{k}$. Clearly, $a_{n} \in \chi\left(x_{n}\right)$. It is easy to check that $\left\|a_{n}\right\|=(1 / n)\left\|y_{n}\right\|+1-1 / n>1$; thus $a_{n} \notin \chi\left(x_{0}\right)$. It is clear that the sequence $\left((1-1 / n) \alpha_{n} e_{n}\right)$ has no accumulation point. Since $(1-1 / n) \alpha_{n} e_{n}=a_{n}-(1 / n) y_{n}$ and $\left(y_{n}\right)$ is bounded, the sequence $\left(a_{n}\right)$ has no accumulation point either. Hence, $U=l^{1} \backslash\left\{a_{n}: n \in \mathbb{N}\right\}$ is open and $\chi\left(x_{0}\right) \subset U$. There is no neighbourhood $V$ of $x_{0}$ such that $\chi(x) \subset U$ for $x \in V$, since $\left(x_{n}\right)$ converges to $x_{0}$. So $\chi$ is not u.s.c. at $x_{0}$.

Let $\mathcal{F}$ denote the topological space $\mathcal{F}_{\mathrm{c}}\left(l^{1}\right)$ with the Vietoris topology. Example 2 shows that the algebraic sum $(F, G) \mapsto F \dot{+} G$ is not a continuous function from $\mathcal{F} \times \mathcal{F}$ to $\mathcal{F}$, since $(\bar{B}(0,1),\{x\}) \mapsto \bar{B}(0,1)+x=\bar{B}(x, 1)$ is not continuous. Similarly, Example 3 shows that multiplication by nonnegative reals $(x, F) \mapsto x F$ is not a continuous function from $[0, \infty) \times \mathcal{F}$ to $\mathcal{F}$, since $(x, \bar{B}(0,1)) \mapsto x \bar{B}(0,1)=\bar{B}(0, x)$ is not continuous. Hence, in the case of the Vietoris topology, a result corresponding to the Rådström-Hörmander Theorem does not hold.

Observe that the Sandwich Theorem does not hold for semicontinuous multifunctions. Indeed, let $\psi: I \rightarrow \mathcal{F}_{\mathrm{c}}\left(l^{1}\right)$ be defined by $\psi(x)=\bar{B}(0,1+x)$. Since $\operatorname{dist}(\psi(x), \psi(y))=|x-y|, \psi$ is H-Lipschitzean. So $\psi$ is also l.s.c. and, by Example 3, it is not u.s.c. Let $\varphi: I \rightarrow \mathcal{F}_{\mathrm{c}}\left(l^{1}\right)$ be defined as $\varphi(x)=\bar{B}(0,1)$ if $x \in[0,1)$ and $\varphi(1)=\bar{B}(0,2)$. Of course, $\varphi$ is u.s.c. and $\varphi(x) \subset \psi(x)$ for every $x \in I$. But, by Example 3, there is no $\mathrm{V}$-continuous $\chi$ such that $\varphi(x) \subset \chi(x) \subset \psi(x)$ for $x \in I$.

Acknowledgements. This research was supported by the Silesian University Mathematics Department (Continuity and Measurability Properties of Multifunctions program). 


\section{References}

[1] C. M. Aseev, Approximation of semicontinuous multivalued mappings by continuous ones, Izv. Akad. Nauk SSSR Ser. Mat. 46 (1982), 460-476 (in Russian); English transl.: Math. USSR-Izv. 20 (1983), 435-448.

[2] C. Castaing and M. Valadier, Convex Analysis and Measurable Multifunctions, Lecture Notes in Math. 580, Springer, Berlin, 1977.

[3] M. M. Čoban and D. M. Ipate, Approximation of multivalued mappings by continuous mappings, Serdica 17 (1991), 127-136 (in Russian).

[4] F. S. De Blasi, Characterizations of certain classes of semicontinuous multifunctions by continuous approximations, J. Math. Anal. Appl. 106 (1985), 1-18.

[5] F. S. De Blasi and G. Pianigiani, Remarks on Hausdorff continuous multifunctions and selections, Comment. Math. Univ. Carolin. 24 (1983), 553-561.

[6] J. Dugundji and A. Granas, Fixed Point Theory, PWN, Warszawa, 1982.

[7] R. Engelking, General Topology, PWN, Warszawa, 1977.

[8] K. M. Garg, On the classification of set-valued functions, Real Anal. Exchange 9 (1983-84), 86-93.

[9] -, A general nonseparable theory of functions and multifunctions, ibid., 317-335.

[10] A. Gavioli, Approximation from the exterior of a multifunction and its application in the "sweeping process", J. Differential Equations 92 (1991), 373-383.

[11] L. Hörmander, Sur la fonction d'appui des ensembles convexes dans un espace localement convexe, Ark. Mat. 3 (1954), 181-186.

[12] A. Kucia, Remarks on intersections of semicontinuous multifunctions, in preparation.

[13] E. Michael, Continuous selections I, Ann. of Math. 63 (1956), 361-382.

[14] H. Rådström, An embedding theorem for spaces of convex sets, Proc. Amer. Math. Soc. 3 (1952), 165-169.

[15] D. Repovš and P. V. Semenov, Continuous Selections of Multivalued Mappings, Kluwer, Dordrecht, 1998.

[16] A. A. Tolstonogov, A theorem on the extension of continuous multivalued maps and its applications, Mat. Zametki 42 (1987), 581-593 (in Russian); English transl.: Math. Notes 42 (1987), 821-827.

[17] R. Urbański, A generalization of the Minkowski-Rådström-Hörmander Theorem, Bull. Acad. Polon. Sci. Sér. Sci. Math. Astronom. Phys. 24 (1976), 709-715.

Anna Kucia

Mathematics Department

Silesian University

Bankowa 14

40-007 Katowice, Poland

E-mail: kucia@ux2.math.us.edu.pl

Received April 7, 2005;

received in final form August 23, 2005 